\section{Associação entre consumo de refrigerantes, sucos e leite, com o índice de massa corporal em escolares da rede pública de Niterói, Rio de Janeiro, Brasil}

\author{
Association between consumption of soft drinks, \\ fruit juice, and milk and body mass index among \\ public school students in Niterói, Rio de Janeiro \\ State, Brazil
}

Fernanda de Albuquerque Melo Nogueira 1 Rosely Sichieri 1

\section{Introdução}

The association between consumption of soft drinks, fruit juice, and milk and body mass index (BMI) was evaluated in 1,423 students 9 to 16 years of age from public schools in Niterói, Rio de Janeiro State, Brazil. Beverage intake was measured using 24-hour recall and a food frequency questionnaire. Weight and height were measured to calculate BMI. Regression analyses took into account the cluster (classes) effect. Analyses were stratified by gender and adjusted for physical activity and age. The results showed a positive association between soft drink intake and age ( $p$ $=0.05$ ) and a negative association between milk and age ( $p=0.004)$. For girls only, there was a significant association between frequent fruit juice intake and BMI ( $\beta=0.02 ; p=0.03)$. For the other beverages, there were no significant associations between BMI and frequent consumption in either gender. Soft drinks and juices accounted for $20 \%$ of mean daily energy intake. The results showed that efforts to reduce energy intake from beverages should include consumption of fruit juice.

Beverages; Drinking; Nutritional Status
Nos Estados Unidos, estudos populacionais conduzidos no período de 1999 a 2002 revelaram que aproximadamente $1 / 3$ dos adultos são obesos ( $27,6 \%$ no sexo masculino e $33,2 \%$ no feminino) e um em cada seis crianças e adolescentes tem sobrepeso ${ }^{1}$. Tal situação é semelhante nos demais países desenvolvidos e na América Latina, onde inquéritos populacionais indicam uma tendência de aumento na prevalência de obesidade não apenas na população adulta, mas também entre crianças e adolescentes 2,3,4,5,6.

No Brasil, dados de pesquisas nacionais identificaram uma tendência de aumento na prevalência de sobrepeso entre crianças e adolescentes. $\mathrm{O}$ inquérito mais recente mostrou que o sobrepeso entre os adolescentes brasileiros triplicou desde 1980, alcançando 17\% em 20037.

A obesidade na adolescência tende a persistir na idade adulta e está associada a graves complicações de saúde 8 . Entre os fatores associados ao aumento da obesidade em crianças e adolescentes, o consumo de bebidas açucaradas tem sido bastante estudado na literatura internacional, com várias pesquisas indicando uma associação positiva entre consumo destas bebidas e ganho de peso 9,10,11,12,13. No Brasil, os dados mais recentes da Pesquisa de Orçamentos Familiares (POF 2002-2003) revelaram que o consumo de refrigerantes aumentou em até 400\%, na população, no período de 1975-2003 14 . 
Apesar desses dados serem preocupantes, a associação entre consumo de bebidas açucaradas e obesidade na adolescência tem sido pouca explorada no país.

Somente um estudo 15 , na cidade de Pelotas, Sul do Brasil, com 607 escolares entre 8 e 10 anos de idade, demonstrou associação significante entre o comportamento alimentar inadequado, caracterizado pela ausência do café da manhã e baixo consumo de leite, e obesidade. Entretanto, quanto ao consumo de refrigerantes, não houve diferenças estatisticamente significantes entre obesos e não-obesos.

Dados da literatura também indicam que o aumento do consumo de refrigerantes tem reduzido o consumo de leite 16.

O objetivo do estudo foi avaliar a associação entre o consumo de bebidas açucaradas e leite, com o índice de massa corporal em escolares.

\section{Métodos}

\section{População de estudo}

Este estudo é parte de um projeto de pesquisa com estudantes matriculados na $4^{\text {a }}$ série de escolas municipais e estaduais do Município de Niterói, no Rio de Janeiro, Brasil, que avaliou a eficácia de um programa de intervenção para prevenção de sobrepeso por meio do estímulo à redução do consumo de refrigerantes 17 . Foram avaliados no presente estudo os dados da linha de base que inclui escolares de 9 a 16 anos de idade.

\section{Desenho de estudo e tamanho da amostra}

Trata-se de um estudo transversal com escolas públicas municipais e estaduais de Niterói, no início do ano letivo de 2005. O tamanho da amostra planejado em 600 participantes permitiria avaliar uma diferença de consumo de 0,5 copo de refrigerante e diferenças de uma unidade no índice de massa corporal (IMC), já levando em conta o desenho do conglomerado (turmas) e prevendo uma taxa de concordância dos pais de $80 \%$. Os dirigentes das escolas não concordaram em selecionar apenas uma única turma de $4 \underline{a}$ ou 5a série para participar do estudo, assim sendo, todas as classes de 4 a série no estado e $5 \underline{a}$ série no município das escolas sorteadas foram incluídas, avaliando-se mais de mil escolares. Com esse tamanho de amostra seria possível estimar com um erro tipo I de $5 \%$ e um erro tipo II de $80 \%$ tanto a prevalência de sobrepeso da ordem de $15 \% 18$ quanto a prevalência de consumo de leite, estimada em aproximadamente $40 \% 19$.
Os pais ou responsáveis pelos estudantes receberam informações sobre os objetivos e procedimentos de coleta dos dados da pesquisa, e somente aqueles que tiveram o seu consentimento assinado pelos responsáveis participaram do estudo.

Do total de escolares matriculados nas turmas escolhidas para a pesquisa $(n=1.509), 98,4 \%$ ( $n=1.485$ ) concordaram em participar do estudo. As demais perdas decorreram da ausência do aluno no dia de coleta de dados e da exclusão de uma criança com 17 anos $(n=62)$, resultando em 1.423 participantes. Desses, 1.216 fizeram antropometria e 1.423 responderam ao questionário.

\section{Coleta de dados}

O consumo de alimentos e de bebidas foi avaliado por um recordatório alimentar de 24 horas e um questionário de freqüência de consumo de bebidas. Esse questionário foi elaborado com base no questionário, previamente validado, para avaliação do consumo alimentar de adultos (QFA) 20. Foram utilizados apenas os itens referentes às bebidas. O questionário incluiu tipo e freqüência de bebidas usualmente consumidas. Para análise, uma porção usual de bebida foi padronizada em um copo de $250 \mathrm{~mL}$ para todas as bebidas avaliadas. As bebidas selecionadas foram: suco de frutas (natural da fruta, industrializado líquido para ser diluído, industrializado pronto para o consumo e industrializado em pó para reconstituição); refrigerante e guaraná natural (light/ diet e normal); leite (desnatado, semidesnatado e integral) e iogurte (light/diet e integral). As freqüências de consumo incluíram oito possibilidades de resposta: nunca ou menos de 1 vez por mês, 1-3 vezes por mês, 1 vez por semana, 2 -4 vezes por semana, 5-6 vezes por semana, 1 vez por dia, $2-3$ vezes por dia e $>3$ vezes por dia. Foi realizado um pré-teste do questionário de freqüência de consumo de bebidas a fim de se avaliar a clareza objetividade das questões e facilidade de aplicação do instrumento.

Ambos os questionários de consumo foram aplicados por cinco nutricionistas previamente treinadas e após a realização das medidas antropométricas.

Embora o controle de qualidade e a avaliação de dados ausentes nos questionários fossem realizados logo após o preenchimento, todos os alunos de uma classe eram liberados em conjunto e alguns tinham mais dificuldades em responder adequadamente às perguntas, não permanecendo por mais tempo disponíveis para a checagem dos questionários (aproximadamente 13\%).

Os dados coletados foram duplamente digitados usando-se o programa Epi Info versão 6.04 
(Centers for Disease Control and Prevention, Atlanta, Estados Unidos). Foram estabelecidos mecanismos de restrição de entrada de dados de maneira a evitar possíveis erros de digitação.

As medidas antropométricas de peso e estatura foram aferidas na escola, com os estudantes utilizando roupas leves, descalços e sem portar objetos pessoais pesados. Todas as medidas foram coletadas por nutricionistas treinados, por meio de procedimentos padronizados 21 . Foi utilizado estadiômetro, com escala de 0 a $200 \mathrm{~cm}$, precisão de $0,1 \mathrm{~cm}$, para medição da estatura (modelo compacto, Wiso, São Paulo, Brasil). A medição foi realizada duas vezes, com os escolares descalços e com a nuca, as nádegas e os calcanhares encostados à parede, sem rodapé, e com a cabeça no plano horizontal de Frankfurt. O valor médio das duas medições foi utilizado nas análises, sendo aceita variação de até $0,5 \mathrm{~cm}$ entre as duas medidas. Se ultrapassado esse valor, as duas medidas eram repetidas. A massa corporal $(\mathrm{kg})$ foi obtida uma única vez, por balança digital, portátil, com capacidade máxima de $150 \mathrm{~kg}$ e precisão de $100 \mathrm{~g}$ (modelo BC 552, Tanita, São Paulo, Brasil).

Para a classificação do estado nutricional dos adolescentes foi calculado o IMC, obtido por meio da divisão do peso em quilogramas pelo quadrado da estatura em metros, sendo utilizada a classificação proposta por Cole et al. 22,23.

O nível de atividade física foi mensurado por meio de duas perguntas: (1) "No recreio você passa a maior parte do tempo brincando/correndo/ jogando bola ou fica sentado/parado?” e (2) “Você pratica exercício físico regular por no mínimo duas vezes por semana fora da escola?" (sim/não).

\section{Análise estatística dos dados}

As freqüências relatadas de consumo das bebidas foram transformadas em oito categorias de freqüências de consumo diárias. Portanto, de 1-3 vezes por mês foi transformada em 0,07 por dia e assim sucessivamente. Para apresentação descritiva em tabelas e respectivos testes de tendência, reagrupou-se em uma menor quantidade de categorias de forma a garantir um número adequado de crianças ( $>10$ ) em todas as caselas. Esses agrupamentos estão descritos nas tabelas.

O teste qui-quadrado foi utilizado para comparar as prevalências de sobrepeso e obesidade por sexo, as proporções entre os tipos de bebidas consumidas segundo o sexo e as prevalências de freqüências de consumo de bebidas por faixa etária.

O teste $\mathrm{t}$ de Student foi realizado para avaliar as diferenças de médias de IMC, peso, altura e idade entre os sexos.
A análise de variância (ANOVA) testou as diferenças das médias de IMC, segundo as categorias de freqüência usual de consumo de bebidas e tipos de bebidas. Foi aplicado o teste de comparações múltiplas de Duncan para determinar quais categorias eram diferentes.

Para avaliar o efeito da freqüência de consumo de bebidas (exposição principal) em relação ao IMC (desfecho), utilizou-se a técnica de regressão linear múltipla, considerando as freqüências de consumo diárias. As análises de regressão foram estratificadas por sexo, ajustadas por idade e considerado o efeito entre conglomerados (turmas) usando-se o procedimento proc surveyreg no programa estatístico SAS (SAS Inst., Cary, Estados Unidos). Como a associação entre IMC e atividade física é inversa e a realização desta atividade pode estimular o consumo de líquidos, as variáveis de atividade física foram posteriormente adicionadas como variáveis de ajuste no modelo 24,25 .

Transformações logarítmicas foram necessárias para normalizar as distribuições da variável IMC. Para avaliação da adequação do modelo final de regressão, realizou-se a análise de resíduos.

Todas as análises estatísticas foram feitas com o programa SAS versão 9.1 .

\section{Resultados}

As prevalências de baixo peso, sobrepeso e obesidade na população estudada, foram $12,1 \%$, $14,8 \%$ e $4,4 \%$, respectivamente. Não houve diferenças estatisticamente significativas nas prevalências de sobrepeso e obesidade entre os sexos ( $\mathrm{p}=0,36$ ). Nos meninos, as prevalências de sobrepeso e obesidade foram de $14,6 \%$ e $3,4 \%$, e nas meninas de $16 \%$ e $5,2 \%$, respectivamente.

As médias de peso, altura e índice de massa corporal foram maiores nas meninas que nos meninos, enquanto que a média de idade foi maior no sexo masculino $(\mathrm{p}<0,05)$.

A maioria dos escolares estudados referiu consumir bebidas integrais ou não dietéticas, sendo encontrada alta prevalência de consumo de refrigerantes não dietéticos $(90,8 \%)$, leite e iogurte integrais $(86,7 \%$ e $81,3 \%$, respectivamente). Houve diferenças entre as médias de IMC segundo os tipos de refrigerantes ( $p<0,0001)$, leite $(\mathrm{p}<0,0001)$ e iogurte $(\mathrm{p}=0,007)$ consumidos, sendo que os valores mais elevados de IMC ocorreram entre os estudantes que referiram consumir produtos light/diet. Não houve diferenças estatisticamente significativas entre as médias de IMC segundo os tipos de sucos consumidos (Tabela 1). 
Prevalências de consumo das bebidas referidas (\%), média do índice de massa corporal (IMC) e desvio-padrão (DP), dos estudantes de 9 a 16 anos, de 22 escolas públicas do Município de Niterói, Rio de Janeiro, Brasil, 2005.

\begin{tabular}{|c|c|c|c|c|}
\hline \multirow[t]{2}{*}{ Bebidas } & \multirow[t]{2}{*}{$\%$} & \multicolumn{3}{|c|}{ IMC } \\
\hline & & Média & DP & Valor de $p$ * \\
\hline Sucos $(n=1.035)$ & & & & 0,49 \\
\hline Industrializado em pó, para ser reconstituído & 41,3 & 17,88 & 1,19 & \\
\hline Industrializado, pronto para o consumo & 2,6 & 18,03 & 1,17 & \\
\hline Industrializado, líquido para ser diluído & 23,0 & 18,26 & 1,19 & \\
\hline Natural da fruta & 28,4 & 18,14 & 1,18 & \\
\hline Não toma & 4,7 & 18,45 & 1,19 & \\
\hline Refrigerantes $(n=1.026)$ & & & & $<0,0001$ \\
\hline Normal & 90,8 & 17,88 & 1,18 & \\
\hline Light/Diet & 3,7 & 19,10 & 1,18 & \\
\hline Normal e light & 5,5 & 20,79 & 1,23 & \\
\hline Leites $(n=1.038)$ & & & & $<0,0001$ \\
\hline Integral & 86,7 & 17,91 & 1,18 & \\
\hline Semidesnatado & 0,7 & 19,94 & 1,21 & \\
\hline Desnatado & 6,4 & 20,26 & 1,26 & \\
\hline Não toma & 6,3 & 18,00 & 1,19 & \\
\hline logurtes $(n=1.030)$ & & & & 0,007 \\
\hline Integral & 81,3 & 17,93 & 1,18 & \\
\hline Light/Diet & 2,3 & 19,57 & 1,19 & \\
\hline Não toma & 16,4 & 18,49 & 1,16 & \\
\hline
\end{tabular}

* ANOVA.

Quanto às freqüências usuais relatadas de consumo de bebidas, verificou-se que tanto para os meninos quanto para as meninas não houve diferenças estatisticamente significantes, com exceção do leite, em que a freqüência foi maior para os meninos, na última categoria. Observouse que refrigerante, suco e guaraná natural são consumidos regularmente, sendo os maiores percentuais encontrados na categoria de $2-4$ vezes por semana. Já para o iogurte, aproximadamente $52 \%$ dos adolescentes referiram consumilo nunca ou quase nunca (Tabela 2).

A Tabela 3 apresenta a associação entre a freqüência de consumo de bebidas e faixa etária. Verificou-se que ocorreu associação apenas entre as categorias de freqüência de consumo de refrigerantes e leite com as faixas etárias, sendo que quanto maior a faixa etária, maior a freqüência de consumo de refrigerantes e menor a de consumo de leite $(\mathrm{p}<0,05)$.

No que diz respeito à média de IMC segundo o tipo e freqüências de consumo de bebidas (Tabela 4), verificou-se que somente nas meninas o valor médio do IMC foi diferente entre as freqüências de consumo de suco $(p=0,03)$. Para as outras bebidas não houve diferenças entre as médias de IMC por freqüências de consumo, em ambos os sexos ( $p>0,05)$.

Como foi encontrada associação positiva entre freqüência de consumo de sucos e IMC, exploraram-se os distintos tipos de sucos ingeridos. Para isso, foram realizados três modelos de regressão múltipla: o modelo 1 avaliou a freqüência de consumo de todos os sucos (industrializado líquido para ser diluído, industrializado pronto para o consumo e industrializado em pó para reconstituição e natural) em relação ao IMC, o modelo 2 avaliou apenas as freqüências de consumo de sucos industrializados excluindo da análise os escolares que referiram tomar suco natural, e o modelo 3 analisou somente o consumo de suco natural, excluindo aqueles que reportaram consumo de suco industrializado. A Tabela 5 apresenta os coeficientes de regressão linear para o IMC, segundo os três modelos de regressão múltipla realizados. Verificou-se que entre as meninas, para cada aumento na freqüência usual de consumo de sucos em geral, ocorreram incrementos na ordem de $0,02 \mathrm{~kg} / \mathrm{m}^{2}$ no IMC, sendo este aumento estatisticamente significante $(\mathrm{p}=$ $0,03)$. Essa associação positiva persiste para sucos industrializados e IMC nas meninas $(\beta=0,03$ 
Tabela 2

Freqüência usual de consumo por tipos de bebidas consumidas, segundo sexo, em estudantes de 9 a 16 anos, de 22 escolas públicas do Município de Niterói, Rio de Janeiro, Brasil, 2005.

\begin{tabular}{|c|c|c|c|c|c|c|c|c|c|c|}
\hline \multirow[t]{3}{*}{ Freqüências de consumo } & \multicolumn{10}{|c|}{ Bebidas consumidas (\%) } \\
\hline & \multicolumn{2}{|c|}{ Suco de fruta } & \multicolumn{2}{|c|}{ Refrigerante } & \multicolumn{2}{|c|}{ Guaraná natural } & \multicolumn{2}{|c|}{ Leite } & \multicolumn{2}{|c|}{ logurte } \\
\hline & $\begin{array}{l}\text { Meninos } \\
(n=591)\end{array}$ & $\begin{array}{l}\text { Meninas } \\
(n=638)\end{array}$ & $\begin{array}{l}\text { Meninos } \\
(n=601)\end{array}$ & $\begin{array}{l}\text { Meninas } \\
(n=646)\end{array}$ & $\begin{array}{l}\text { Meninos } \\
(n=602)\end{array}$ & $\begin{array}{l}\text { Meninas } \\
(n=646)\end{array}$ & $\begin{array}{l}\text { Meninos } \\
(n=597)\end{array}$ & $\begin{array}{l}\text { Meninas } \\
(n=636)\end{array}$ & $\begin{array}{l}\text { Meninos } \\
(n=603)\end{array}$ & $\begin{array}{l}\text { Meninas } \\
(n=647)\end{array}$ \\
\hline Nunca ou quase nunca & 5,1 & 6,7 & 9,8 & 12,1 & 29,7 & 10,4 & 10,4 & 9,0 & 52,2 & 52,2 \\
\hline 1 vez por semana & 10,7 & 11,0 & 8,6 & 8,4 & 6,0 & 3,4 & 4,7 & 3,6 & 1,7 & 1,7 \\
\hline $2-4$ vezes por semana & 43,1 & 42,2 & 39,9 & 42,2 & 30,7 & 29,1 & 16,9 & 21,9 & 22,1 & 22,7 \\
\hline $5-6$ vezes por semana & 9,6 & 9,7 & 18,3 & 18,0 & 18,9 & 19,8 & 4,7 & 6,0 & 16,7 & 14,8 \\
\hline 1 vez por dia & 12,0 & 9,7 & 8,3 & 6,3 & 9,6 & 10,8 & 25,6 & 29,6 & 5,0 & 5,9 \\
\hline$\geq 2$ vezes por dia & 19,5 & 20,7 & 15,0 & 13,0 & 5,0 & 3,4 & 37,7 & 30,0 & 2,3 & 2,6 \\
\hline Valor de $p$ * & \multicolumn{2}{|c|}{0,99} & \multicolumn{2}{|c|}{0,13} & \multicolumn{2}{|c|}{0,08} & \multicolumn{2}{|c|}{0,02} & \multicolumn{2}{|c|}{0,48} \\
\hline
\end{tabular}

* Teste do qui-quadrado para comparação entre os sexos.

Tabela 3

Freqüência de bebidas usualmente consumidas (\%), segundo a faixa etária, por estudantes de 22 escolas públicas do Município de Niterói, Rio de Janeiro, Brasil, 2005.

\begin{tabular}{|c|c|c|c|}
\hline \multirow[t]{2}{*}{ Bebida } & \multicolumn{3}{|c|}{ Faixa etária (anos) } \\
\hline & $9,0-10,9$ & $11,0-12,9$ & $\geq 13,0$ \\
\hline \multicolumn{4}{|l|}{ Refrigerante $(n=1.243)$} \\
\hline Nunca ou quase nunca & 10,8 & 11,4 & 8,8 \\
\hline 1-6 vezes por semana & 69,1 & 67,1 & 63,7 \\
\hline 1 vez por dia & 7,6 & 6,7 & 6,9 \\
\hline$\geq 2$ vezes por dia & 12,4 & 14,8 & 20,6 \\
\hline Valor de $p$ & & 0,05 & \\
\hline \multicolumn{4}{|l|}{ Leite $(n=1.229)$} \\
\hline Nunca ou quase nunca & 7,7 & 9,8 & 21,6 \\
\hline 1-6 vezes por semana & 27,7 & 30,0 & 32,3 \\
\hline 1 vez por dia & 30,0 & 25,5 & 21,6 \\
\hline$\geq 2$ vezes por dia & 34,5 & 34,7 & 24,5 \\
\hline Valor de $\mathrm{p}$ & & 0,004 & \\
\hline \multicolumn{4}{|l|}{ logurte $(n=1.246)$} \\
\hline Nunca ou quase nunca & 51,3 & 52,6 & 55,9 \\
\hline 1-6 vezes por semana & 40,7 & 39,2 & 38,2 \\
\hline 1 vez por dia & 6,0 & 4,8 & 4,9 \\
\hline$\geq 2$ vezes por dia & 2,1 & 3,4 & 1,0 \\
\hline Valor de $\mathrm{p}$ & & 0,56 & \\
\hline \multicolumn{4}{|l|}{ Sucos $(n=1.225)$} \\
\hline Nunca ou quase nunca & 6,3 & 5,3 & 6,7 \\
\hline 1-6 vezes por semana & 62,9 & 64,1 & 59,8 \\
\hline 1 vez por dia & 11,1 & 10,3 & 11,8 \\
\hline$\geq 2$ vezes por dia & 19,7 & 20,3 & 21,6 \\
\hline Valor de $p$ & & 0,66 & \\
\hline \multicolumn{4}{|l|}{ Guaraná natural $(n=1.244)$} \\
\hline Nunca ou quase nunca & 32,2 & 31,9 & 25,5 \\
\hline 1-6 vezes por semana & 52,8 & 55,1 & 56,9 \\
\hline 1 vez por dia & 10,2 & 10,3 & 10,8 \\
\hline$\geq 2$ vezes por dia & 4,8 & 2,7 & 6,9 \\
\hline Valor de $\mathrm{p}$ & & 0,69 & \\
\hline
\end{tabular}


Tabela 4

Média do índice de massa corporal (IMC), segundo a freqüência usual de consumo de bebidas por sexo, em estudantes de 9 a 16 anos, de 22 escolas públicas do Município de Niterói, Rio de Janeiro, Brasil, 2005.

\begin{tabular}{|c|c|c|c|c|c|c|c|c|c|c|}
\hline \multirow[t]{2}{*}{ Freqüência usual } & \multicolumn{2}{|c|}{$\begin{array}{l}\text { Suco de fruta } \\
(n=541)\end{array}$} & \multicolumn{2}{|c|}{$\begin{array}{l}\text { Refrigerante } \\
\qquad(n=548)\end{array}$} & \multicolumn{2}{|c|}{$\begin{array}{l}\text { Guaraná natural } \\
\qquad(n=548)\end{array}$} & \multicolumn{2}{|c|}{$\begin{array}{c}\text { Leite } \\
(n=538)\end{array}$} & \multicolumn{2}{|c|}{$\begin{array}{l}\text { logurte } \\
(n=549)\end{array}$} \\
\hline & IMC & DP & IMC & DP & IMC & DP & IMC & DP & IMC & DP \\
\hline \multicolumn{11}{|l|}{ Meninas } \\
\hline Nunca ou quase nunca & 17,03 * & 1,16 & 18,70 & 1,21 & 18,49 & 1,22 & 17,46 & 1,20 & 18,34 & 1,21 \\
\hline 1 vez por semana & 18,60 & 1,15 & 18,86 & 1,21 & 18,25 & 1,17 & 18,89 & 1,20 & 17,89 & 1,18 \\
\hline 2-4 vezes por semana & 18,10 & 1,19 & 18,27 & 1,21 & 18,26 & 1,20 & 18,28 & 1,17 & 18,36 & 1,20 \\
\hline $5-6$ vezes por semana & 18,46 & 1,18 & 17,67 & 1,19 & 17,98 & 1,19 & 17,79 & 1,22 & 18,60 & 1,09 \\
\hline 1 vez por dia & 17,70 & 1,19 & 17,22 & 1,17 & 17,62 & 1,20 & 18,71 & 1,21 & 18,35 & 1,19 \\
\hline$\geq 2$ vezes por dia & 18,94 * & 1,25 & 18,00 & 1,16 & 18,05 & 1,22 & 18,02 & 1,21 & 17,54 & 1,12 \\
\hline Valor de $p$ & \multicolumn{2}{|c|}{0,03} & \multicolumn{2}{|c|}{0,08} & \multicolumn{2}{|c|}{0,64} & \multicolumn{2}{|c|}{0,16} & \multicolumn{2}{|c|}{0,83} \\
\hline Valor de $\mathrm{p}$ da tendência & \multicolumn{2}{|c|}{0,02} & \multicolumn{2}{|c|}{0,06} & \multicolumn{2}{|c|}{0,15} & \multicolumn{2}{|c|}{0,77} & \multicolumn{2}{|c|}{0,63} \\
\hline \multicolumn{11}{|l|}{ Meninos } \\
\hline Nunca ou quase nunca & 17,21 & 1,12 & 17,74 & 1,16 & 17,75 & 1,18 & 18,68 & 1,21 & 17,80 & 1,19 \\
\hline $1 \mathrm{vez}$ por semana & 17,31 & 1,16 & 18,10 & 1,15 & 17,87 & 1,18 & 17,52 & 1,17 & 17,95 & 1,16 \\
\hline $2-4$ vezes por semana & 17,85 & 1,17 & 17,82 & 1,18 & 17,89 & 1,16 & 17,92 & 1,18 & 17,79 & 1,18 \\
\hline $5-6$ vezes por semana & 18,05 & 1,16 & 18,00 & 1,21 & 17,98 & 1,21 & 17,42 & 1,14 & 18,47 & 1,08 \\
\hline 1 vez por dia & 18,49 & 1,20 & 18,12 & 1,20 & 17,76 & 1,16 & 17,95 & 1,19 & 17,40 & 1,10 \\
\hline$\geq 2$ vezes por dia & 17,75 & 1,18 & 17,50 & 1,17 & 18,04 & 1,22 & 17,62 & 1,15 & 19,34 & 1,16 \\
\hline Valor de p & \multicolumn{2}{|c|}{0,29} & \multicolumn{2}{|c|}{0,81} & \multicolumn{2}{|c|}{0,99} & \multicolumn{2}{|c|}{0,31} & \multicolumn{2}{|c|}{0,54} \\
\hline Valor de $\mathrm{p}$ da tendência & \multicolumn{2}{|c|}{0,50} & \multicolumn{2}{|c|}{0,36} & \multicolumn{2}{|c|}{0,70} & \multicolumn{2}{|c|}{0,11} & \multicolumn{2}{|c|}{0,30} \\
\hline
\end{tabular}

* Médias que diferiram significativamente após o teste de comparações múltiplas (teste de Duncan, p $<0,05$ ).

Tabela 5

Coeficiente de regressão linear para o índice de massa corporal (IMC) segundo a freqüência de consumo de sucos, em modelos ajustados por idade e considerando o efeito do conglomerado.

\begin{tabular}{lcccc}
\hline Modelos & $\mathbf{n}$ & $\beta$ & Valor de $\mathbf{p}$ & $\mathbf{R}$ (\%) \\
\hline Meninas & & & & \\
1. Sucos em geral & 541 & 0,02 & 0,03 & 5,2 \\
2. Suco industrializado & 368 & 0,03 & 0,06 & 5,0 \\
3. Suco 100\% natural & 149 & 0,03 & 0,18 & 0,1 \\
Meninos & & & 0,48 \\
1. Sucos em geral & 484 & 0,008 & 0,06 \\
2. Suco industrializado & 324 & 0,002 & 0,90 & 1,12 \\
3. Suco 100\% natural & 149 & $-0,006$ & 0,76 & \\
\hline
\end{tabular}

$\left.\mathrm{p}=0,06, \mathrm{R}^{2}=6,0 \%\right)$. Já na categoria de sucos naturais, não foi verificada associação significante com o IMC. Após a inclusão de duas variáveis de atividade física no modelo, os resultados mantiveram-se inalterados.

Realizou-se a análise de resíduo para avaliar a adequação da modelagem estatística, bem como a análise adicional, com exclusão de três valo- res outliers, sem alterações nas estimativas das exposições principais, indicando que o modelo estava bem ajustado.

Analisando o recordatório alimentar dos adolescentes avaliados, a média de consumo de energia foi de $2.437 \pm 998 \mathrm{kcal}$ para os meninos, e de $2.190 \pm 950 \mathrm{kcal}$ para as meninas. Sendo que para os primeiros, os refrigerantes contri- 
buíram com cerca de 8,8\% (214 $\pm 159 \mathrm{kcal})$, sucos industrializados com $7 \%(169 \pm 100 \mathrm{kcal}) \mathrm{e}$ sucos $100 \%$ naturais com $1,9 \%$ (48 $\pm 49 \mathrm{kcal})$ do total de calorias ingeridas. Já para as meninas, os refrigerantes contribuíram com cerca de 9,9\% ( $216 \pm 141 \mathrm{kcal})$, sucos industrializados com $6,4 \%$ (139 $\pm 78 \mathrm{kcal})$ e sucos $100 \%$ naturais com $1,9 \%$ ( $43 \pm 46 \mathrm{kcal}$ ) do total de calorias ingeridas. Houve diferenças estatisticamente significantes, entre os sexos, na média de calorias totais ingeridas e nas calorias provenientes do consumo de sucos industrializados $(\mathrm{p}<0,05)$.

\section{Discussão}

A análise do consumo de bebidas pelo QFA revelou que aproximadamente $20 \%$ dos escolares avaliados referiram o consumo diário, e $60 \%$ semanal ( 2 a 6 vezes por semana) de refrigerantes e sucos. Esse consumo pode representar quase $20 \%$ do consumo diário de energia, quando se analisou a contribuição energética das bebidas usando-se o método recordatório de 24 horas. Elevadas prevalências no consumo de bebidas açucaradas também foram encontradas no estudo conduzido por Bere et al. 26 , com 2.870 adolescentes da Noruega, sendo que $63 \%$ consumiram refrigerantes duas vezes por semana ou mais. $\mathrm{O}$ estudo realizado porWang et al. 27, em uma amostra representativa de adolescentes americanos, entre 12 e 19 anos de idade, que participaram do National Health and Nutrition Examination Survey (NHANES: 1988-1994 e 1999-2004) revelou que a ingestão de bebidas açucaradas e de sucos aumentou ao longo destes anos, contribuindo com cerca $13 \%$ (341kcal) e 1,9\% (43kcal), respectivamente, do consumo médio diário de energia, no último período estudado. É provável que o alto consumo de bebidas açucaradas decorra da propaganda indiscriminada destes produtos que são lançados no mercado, atingindo facilmente os domicílios e instituições de ensino, onde há uma aglomeração de crianças e adolescentes, estimulando o seu consumo nesta faixa etária 28 . Além disso, o baixo preço dessas bebidas associado ao gosto agradável também são fatores que incentivam o seu consumo 29.

No presente estudo, embora 93\% dos estudantes tenham referido ingerir algum tipo de leite, menos de $40 \%$ deles relataram consumi-lo duas vezes por dia ou mais. Esse achado é preocupante uma vez que a necessidade de cálcio dietético nessa faixa etária é alta e recomendase a ingestão de três porções de leite magro ou derivados, diariamente, visando à promoção da densidade mineral óssea e crescimento linear adequado durante o período da adolescência, e à prevenção da osteoporose, osteopenia e outras complicações provenientes da deficiência de cálcio na vida adulta 30 .

A prevalência de consumo de refrigerantes foi maior em escolares mais velhos e menor em escolares mais novos, e a prevalência de consumo de leite foi maior em estudantes mais novos e menor nos mais velhos. Esses dados são consistentes com a literatura, que tem revelado uma tendência de substituição da ingestão de leite por refrigerante com o aumento da idade 31,32,33. Cabe ressaltar que em estudo recente, realizado por Dubois et al. ${ }^{34}$, verificou-se que essa tendência também está ocorrendo em pré-escolares. Esses autores, ao seguirem uma coorte de 2.103 crianças entre 2 e 5 anos de idade, em Québec, Canadá, verificaram que a proporção de crianças que consomem refrigerante no mínimo uma vez por semana entre as refeições aumentou com a idade ( $42 \%$ aos 2,5 anos; 47 e $48 \%$ aos 3,5 e 4,5 anos, respectivamente.

Quanto à média de IMC, segundo o tipo de bebida ingerida, os valores mais altos ocorreram para os grupos de bebidas diet/light ou com quantidades de gorduras diminuídas. Esse achado é um típico exemplo de causalidade reversa, muito freqüente em estudos transversais. Provavelmente, os escolares com maiores valores de IMC ingerem maiores proporções de bebidas diet/light ou com teor reduzido de gordura para redução de seu peso.

Os dados apresentados em estudos transversais referentes à associação entre consumo de bebidas açucaradas e sobrepeso/obesidade ainda são controversos 13,35. Giammattei et al. 36, ao avaliarem uma amostra probabilística de 385 adolescentes entre 11 e 14 anos de idade, residentes na Califórnia, Estados Unidos, verificaram que a média de IMC, o percentual de gordura corporal total e a prevalência de sobrepeso foram maiores no grupo que consumiu refrigerante três vezes ao dia ou mais, comparados aos que não consumiram ( $\mathrm{p}=0,003, \mathrm{p}=0,02 \mathrm{e} \mathrm{p}=$ 0,006 , respectivamente). Já outros estudos transversais também nos Estados Unidos 33,37,38,39 não identificaram essa associação.

No presente estudo, não foi encontrada associação positiva entre consumo de refrigerante e IMC. Observou-se que o consumo de refrigerantes entre os escolares brasileiros é alto, tão grande quanto em outros países que encontraram essa associação 10,36,40. É possível que a ausência de associação seja uma limitação do próprio desenho da pesquisa, uma vez que em estudos longitudinais essa associação se faz presente 10,34,40.

Deve-se considerar também a possibilidade de ocorrência de viés de informação como uma justificativa para ausência de associação entre 
freqüência de consumo de refrigerante e IMC. Possivelmente, nessa faixa etária, os adolescentes já tenham informação de que refrigerante não é uma bebida saudável, devendo ser consumida o menos possível. Dados do Brasil 41 revelam que indivíduos com sobrepeso ou obesos sub-relatam o consumo alimentar. Na amostra estudada, aqueles que apresentaram valores de IMC mais alto poderiam ter relatado uma freqüência de consumo de refrigerantes não dietéticos mais baixa que a usual, subestimando a freqüência usual de consumo e mascarando o consumo real de refrigerantes neste grupo. Finalmente, não se pode descartar a hipótese de um menor efeito na adiposidade da composição dos refrigerantes fabricados no Brasil quando comparados àqueles fabricados nos Estados Unidos. O refrigerante nacional tem sacarose como única fonte de açúcar, não sendo adicionado de HFCS (hight fructose corn syrup), que está presente em refrigerantes produzidos nos países norte-americanos e que induz resposta hormonal diferenciada, alterando a relação insulina-leptina favorecendo a lipogênese 42 .

Nas meninas, o consumo de sucos associouse significativa e positivamente com o IMC, enquanto que nos meninos não foi encontrada associação. Existe teoricamente a possibilidade de viés de informação em direção à redução do consumo de refrigerantes e aumento do consumo de sucos, uma vez que sucos representam bebidas saudáveis. Considerando que as meninas quando comparadas aos meninos tendem a ser mais atentas às recomendações nutricionais e mais preocupadas com as questões de saúde e, após análise do consumo do recordatório alimentar de 24 horas, que revelou que as meninas consumiram menos calorias provenientes de suco industrializado que os meninos, há a possibilidade de ocorrência de super-relato na freqüência de consumo de sucos referida. Além disso, nesse ciclo da vida, as meninas apresentam alterações hormonais que favorecem o ganho de peso corporal, o que poderia explicar os valores mais elevados de IMC.
O consumo de sucos pela população estudada representa uma fonte adicional e importante de calorias, além das provenientes dos refrigerantes, uma vez que $90 \%$ dos escolares reportaram o consumo de refrigerantes não dietéticos. Os resultados mostraram que o consumo de sucos industrializados representa uma contribuição calórica maior que o de sucos $100 \%$ naturais, e em meninas associou-se positivamente com o IMC. Em análise anterior desses dados, encontrou-se associação positiva entre consumo de açúcar e sucos ( $r=0,35$; $p>0,0001)$ e negativa entre consumo de açúcar e refrigerantes $(r=-0,16$; $\mathrm{p}<0,001$ ), sugerindo que os açúcares são acrescidos durante o preparo de todos os sucos 16 .

Associação positiva entre consumo usual de sucos e sobrepeso/obesidade também foi encontrada no estudo conduzido por Sanigorski et al. 43 , com uma amostra probabilística de 2.184 crianças entre 4 e 12 anos, em Victoria, Austrália. Segundo esses autores, o consumo regular de sucos (duas vezes por dia ou mais) esteve associado positivamente com sobrepeso/obesidade quando comparado ao consumo menor que uma vez por semana. Tanasescu et al. ${ }^{44}$, ao realizarem um estudo caso-controle na cidade de Hartford em Porto Rico, com 53 crianças entre 7 e 11 anos de idade, visando a identificar fatores preditores para a obesidade, observaram que aqueles que consumiram diariamente um porção de sucos de fruta (incluindo sucos aromatizados artificialmente com pouca quantidade ou sem polpa de fruta) apresentaram 4,02 mais chances de se tornar obesos quando comparados aos que não consumiram.

O estudo sugere que esforços para redução de energia por meio de fontes líquidas devam incluir além dos refrigerantes, os sucos, principalmente os industrializados, que são em geral considerados como parte de uma alimentação saudável. Mas, que se tornam veículos importantes de adição de açúcar. 


\section{Resumo}

Avaliou-se a associação entre o consumo de refrigerantes, sucos e leite, com o indice de massa corporal (IMC) em 1.423 estudantes, entre 9 e 16 anos, de escolas públicas de Niterói, Rio de Janeiro, Brasil. O consumo de bebidas foi avaliado por meio do recordatório alimentar de 24 horas e questionário de freqüência de consumo alimentar. Peso e estatura foram coletados para o cálculo do IMC. As análises de regressão linear foram estratificadas por sexo e ajustadas por atividade física, idade e efeito do conglomerado (classes). Verificou-se associação positiva entre freqüência de consumo de refrigerante e idade $(p=0,05)$ e negativa entre consumo de leite e idade $(p=0,004)$. Apenas para as meninas, o IMC associou-se positivamente com o consumo de $\operatorname{sucos}(\beta=0,02 ; p=0,03)$. Para as outras bebidas não foram encontradas associações entre IMC e freqüência usual de consumo. O consumo de refrigerantes e sucos representou cerca de $20 \%$ do total de energia média consumida diariamente. Os resultados indicam que esforços para reduzir a ingestão de energia por meio de bebidas devem enfatizar também os sucos.

Bebidas; Ingestão de Líquidos; Estado Nutricional

\section{Colaboradores}

F. A. M. Nogueira contribuiu substancialmente para a concepção e planejamento, análise e interpretação dos dados, contribuiu significativamente na elaboração do rascunho e participou da aprovação da versão final do manuscrito. R. Sichieri contribuiu na análise e interpretação dos dados, na revisão crítica do conteúdo e aprovação da versão final do manuscrito.

\section{Referências}

1. Baskin ML, Ard J, Franklin F, Allison DB. Prevalence of obesity in the United States. Obes Rev 2005; 6:5-7.

2. Filozof C, Gonzalez C, Sereday M, Mazza C, Braguinsky J. Obesity prevalence and trends in LatinAmerican countries. Obes Rev 2001; 2:99-106.

3. Moraes SA, Beltrán-Rosas J, Mondini L, Freitas ICM. Prevalência de sobrepeso e obesidade e fatores associados em escolares de área urbana de Chilpancingo, Guerrero, México, 2004. Cad Saúde Pública 2006; 22:1289-301.

4. Venn AJ, Thomson RJ, Schmidt MD, Cleland VJ, Curry BA, Gennat HC, et al. Overweight and obesity from childhood to adulthood: a follow-up of participants in the 1985 Australian Schools Health and Fitness Survey. Med J Aust 2007; 186:458-60.

5. Whelton H, Harrington J, Crowley E, Kelleher V, Cronin M, Perry IJ. Prevalence of overweight and obesity on the island of Ireland: results from the North South Survey of Children's Height, Weight and Body Mass Index, 2002. BMC Public Health 2007; 7:187.

6. Willows ND, Johnson MS, Ball GD. Prevalence estimates of overweight and obesity in Cree preschool children in northern Quebec according to international and US reference criteria. Am J Public Health 2007; 97:311-6.
7. Instituto Brasileiro de Geografia e Estatística. Pesquisa de orçamentos familiares 2002-2003: primeiros resultados Brasil e grandes regiões. Rio de Janeiro: Instituto Brasileiro de Geografia e Estatística; 2004

8. Nathan BM, Moran A. Metabolic complications of obesity in childhood and adolescence: more than just diabetes. Curr Opin Endocrinol Diabetes Obes 2008; 15:21-9.

9. Troiano RP, Briefel RR, Carroll MD, Bialostosky K. Energy and fat intakes of children and adolescents in the United States: data from the national health and nutrition examination surveys. Am J Clin Nutr 2000; 72 (5 Suppl):1343S-53.

10. Ludwig DS, Peterson KE, Gortmaker SL. Relation between consumption of sugar-sweetened drinks and childhood obesity: a prospective, observational analysis. Lancet 2001; 357:505-8.

11. Ballew C, Kuester S, Gillespie C. Beverage choices affect adequacy of children's nutrient intakes. Arch Pediatr Adolesc Med 2000; 154:1148-52.

12. Cullen KW, Zakeri I. Fruits, vegetables, milk, and sweetened beverages consumption and access to a la carte/snack bar meals at school. Am J Public Health 2004; 94:463-7. 
13. Vartanian LR, Schwartz MB, Brownell KD. Effects of soft drink consumption on nutrition and health: a systematic review and meta-analysis. Am J Public Health 2007; 97:667-75.

14. Levy-Costa RB, Sichieri R, Pontes NS, Monteiro CA. Disponibilidade domiciliar de alimentos no Brasil: distribuição e evolução (1974-2003). Rev Saúde Pública 2005; 39:530-40.

15. Triches RM, Giugliani ERJ. Obesidade, práticas alimentares e conhecimentos de nutrição em escolares. Rev Saúde Pública 2005; 39:541-7.

16. Cavadini C, Siega-Riz AM, Popkin BM. US adolescent food intake trends 1965 to 1996. Arch Dis Child 2000; 83:18-24.

17. Sichieri R, Trotte AP, Souza RA, Veiga GV. School randomised trial on prevention of excessive weight gain by discouraging students from drinking sodas. Public Health Nutr 2009; 12:197-202.

18. Vieira ACR, Alvarez MM, Marins VMR, Sichieri R, Veiga GV. Desempenho e pontos de corte do índice de massa corporal de diferentes referências na predição de gordura corporal em adolescentes. Cad Saúde Pública 2006; 22:1681-90.

19. Teixeira MH, Veiga GV, Sichieri R. Consumo de gordura e hipercolesterolemia em uma amostra probabilística de estudantes de Niterói, Rio de Janeiro. Arq Bras Endocrinol Metab 2007; 51:65-71.

20. Sichieri R, Everhart JE. Validity of a Brazilian food frequency questionnaire against dietary recalls and estimated energy intake. Nutr Res 1998; 18:1649-59.

21. Lohman T, Roche A, Martorell R. Anthropometric standardization reference manual. Champaign: Human Kinetics; 1988.

22. Cole TJ, Bellizzi MC, Flegal KM, Dietz WH. Establishing a standard definition for child overweight and obesity worldwide: international survey. BM] 2000; 320:1240-3.

23. Cole TJ, Flegal KM, Nicholls D, Jackson AA. Body mass index cut offs to define thinness in children and adolescents: international survey. BMJ 2007; 335:194.

24. Hemmingsson E, Ekelund U. Is the association between physical activity and body mass index obesity dependent? Int J Obes (Lond) 2007; 31:663-8.

25. St-Onge MP, Keller KL, Heymsfield SB. Changes in childhood food consumption patterns: a cause for concern in light of increasing body weights. Am J Clin Nutr 2003; 78:1068-73.

26. Bere E, Glomnes ES, te Velde SJ, Klepp KI. Determinants of adolescents' soft drink consumption. Public Health Nutr 2008; 11:49-56.

27. Wang YC, Bleich SN, Gortmaker SL. Increasing caloric contribution from sugar-sweetened beverages and $100 \%$ fruit juices among US children and adolescents, 1988-2004. Pediatrics 2008; 121: e1604-14.

28. Mello MM, Pomeranz J, Moran P. The interplay of public health law and industry self-regulation: the case of sugar-sweetened beverage sales in schools. Am J Public Health 2008; 98:595-604.

29. Drewnowski A, Monsivais P, Maillot M, Darmon N. Low-energy-density diets are associated with higher diet quality and higher diet costs in French adults. J Am Diet Assoc 2007; 107:1028-32.
30. US Department of Health and Human Services/ US Department of Agriculture. Dietary Advisory Guidelines Committee. Dietary guidelines for Americans. Washington DC: US Department of Health and Human Services/US Department of Agriculture; 2005.

31. Harnack L, Stang J, Story M. Soft drink consumption among US children and adolescents: nutritional consequences. J Am Diet Assoc 1999; 99: 436-41.

32. Mrdjenovic G, Levitsky DA. Nutritional and energetic consequences of sweetened drink consumption in 6- to 13-year-old children. J Pediatr 2003; 142:604-10.

33. Forshee RA, Storey ML. Total beverage consumption and beverage choices among children and adolescents. Int J Food Sci Nutr 2003; 54:297-307.

34. Dubois L, Farmer A, Girard M, Peterson K. Regular sugar-sweetened beverage consumption between meals increases risk of overweight among preschool-aged children. J Am Diet Assoc 2007; 107:924-34.

35. Malik VS, Schulze MB, Hu FB. Intake of sugarsweetened beverages and weight gain: a systematic review. Am J Clin Nutr 2006; 84:274-88.

36. Giammattei J, Blix G, Marshak HH, Wollitzer AO, Pettitt DJ. Television watching and soft drink consumption: associations with obesity in 11- to 13year-old schoolchildren. Arch Pediatr Adolesc Med 2003; 157:882-6.

37. Forshee RA, Anderson PA, Storey ML. The role of beverage consumption, physical activity, sedentary behavior, and demographics on body mass index of adolescents. Int J Food Sci Nutr 2004; 55:463-78.

38. Rajeshwari R, Yang SJ, Nicklas TA, Berenson GS. Secular trends in children's sweetened-beverage consumption (1973 to 1994): the Bogalusa Heart Study. J Am Diet Assoc 2005; 105:208-14.

39. O'Connor TM, Yang SJ, Nicklas TA. Beverage intake among preschool children and its effect on weight status. Pediatrics 2006; 118:e1010-8.

40. Berkey CS, Rockett HR, Field AE, Gillman MW, Colditz GA. Sugar-added beverages and adolescent weight change. Obes Res 2004; 12:778-88.

41. de Salvo VLMA, Gimeno SGA. Reprodutibilidade e validade do questionário de freqüência de consumo de alimentos. Rev Saúde Pública 2002; 36: 5005-12.

42. Bray GA, Nielsen SJ, Popkin BM. Consumption of high-fructose corn syrup in beverages may play a role in the epidemic of obesity. Am J Clin Nutr 2004; 79:537-43.

43. Sanigorski AM, Bell AC, Swinburn BA. Association of key foods and beverages with obesity in Australian schoolchildren. Public Health Nutr 2007; 10:152-7.

44. Tanasescu M, Ferris AM, Himmelgreen DA, Rodriguez N, Perez-Escamilla R. Biobehavioral factors are associated with obesity in Puerto Rican children. J Nutr 2000; 130:1734-42.

Recebido em 09/Jan/2009

Versão final reapresentada em 05/Set/2009

Aprovado em 22/Set/2009 